\title{
Contrasting Cortical Activity Associated with Category Memory and Recognition Memory
} Paul J. Reber, ${ }^{1}$ Craig E.L. Stark, ${ }^{1}$ and Larry R. Squire ${ }^{1-4}$

Departments of ${ }^{1}$ Psychiatry and ${ }^{2}$ Neurosciences

University of California at San Diego

La Jolla, California 92093 USA

${ }^{3}$ Veterans Affairs Medical Center

San Diego, California 92161 USA

\begin{abstract}
We collected functional neuroimaging data while volunteers performed similar categorization and recognition memory tasks. In the categorization task, volunteers first studied a series of 40 dot patterns that were distortions of a nonstudied prototype dot pattern. After a delay, while fMRI data were collected, they categorized 72 novel dot patterns according to whether or not they belonged to the previously studied category. In the recognition task, volunteers first studied five dot patterns eight times each. After a delay, while fMRI data were collected, they judged whether each of 72 dot patterns had been studied earlier. We found strikingly different patterns of brain activity in visual processing areas for the two tasks. During the categorization task, the familiar stimuli were associated with decreased activity in posterior occipital cortex, whereas during the recognition task, the familiar stimuli were associated with increased activity in this area. The findings indicate that these two types of memory have contrasting effects on early visual processing and reinforce the view that declarative and nondeclarative memory operate independently.
\end{abstract}

\section{Introduction}

Long-term memory is composed of multiple separate systems (Squire 1982; Weiskrantz 1990; Schacter and Tulving 1994). Declarative memory is expressed in the conscious recall and recognition

${ }^{4}$ Corresponding author. of past events, whereas nondeclarative memory is expressed through nonconscious changes in performance. Examples of nondeclarative memory include the ability to learn a visual category by studying exemplars derived from a prototype (category learning) and facilitation in the ability to detect and identify recently encountered stimuli (repetition priming). A key question in the study of memory systems has been whether nondeclarative memory phenomena such as priming or category learning influence recognition memory. The two types of memory could operate independently (Squire et al. 1993; Schacter 1994; Wagner et al. 1997; Schacter and Buckner 1998), or recognition memory judgments could be based partly on a feeling of familiarity derived from nondeclarative memory (Mandler 1980, 1989; Jacoby and Dallas 1981; Jacoby 1991; Gardiner and Java 1993).

Functional neuroimaging studies have shown that both visual priming (Squire et al. 1992; Schacter and Buckner 1998; Wiggs and Martin 1998) and visual category learning (Reber et al. 1998a) are associated with decreases in activity in posterior cortical regions. A common account of this finding is that the reduced activity reflects improved perceptual facility for previously encountered visual stimuli. It is not known whether improved perceptual facility contributes to performance in declarative memory tasks such as conventional tests of recognition memory. One technique for addressing this question is to identify whether the reductions in cortical activity that occur in tasks of priming and visual category learning also occur when individuals are making conscious recognition memory judgments.

The task used to study category learning was a modification of one introduced by Posner and Keele (1968) (Knowlton and Squire 1993). Eight volunteers studied a series of 40 dot patterns (Fig. 1) that were distortions of an underlying proto-

LEARNING \& MEMORY 5:420-428 @ 1998 by Cold Spring Harbor Laboratory Press ISSN1072-0502/98 \$5.00

$$
\begin{array}{lllllllllllllll}
L & E & A & R & N & I & N & G & \underset{420}{\boldsymbol{Z}} & M & E & M & O & R & Y
\end{array}
$$


typic dot pattern. Subsequently, they were told that the study items belonged to a single category and that they should now try to classify new dot patterns according to whether or not they belonged to the same category. Functional magnetic resonance imaging (fMRI) data were collected while volunteers performed the dot pattern categorization task with 72 novel patterns, 36 of which belonged to the trained category and $36 \mathrm{did}$ not.

Ten different volunteers received a parallel recognition memory task that also used dot patterns. They first studied 5 distinct dot patterns, each presented eight times in a random order for a total of 40 study patterns. fMRI data were then collected while the volunteers took a recognition memory test containing 36 old patterns (the 5 study patterns were each repeated seven or eight times) and 36 new patterns.

\section{Materials and Methods}

Eighteen healthy, right-handed volunteers (10 women, 8 men; mean age $=25 \mathrm{yr}$ ) gave written informed consent prior to participating in the study. Both the study and test portions of the behavioral paradigm were performed in the MRI scanner, but fMRI data were collected only during the test phase. A mirror was placed so that stimuli could be back-projected onto a viewing screen 3.5 $\mathrm{m}$ from the subject's head. The material on the screen subtended a visual angle of $5^{\circ}-7^{\circ}$.

\section{CATEGORY LEARNING}

Eight volunteers studied 40 dot patterns one at a time (Fig. 1). Each pattern was composed of nine dots, constructed as described previously (Posner and Keele 1968; Knowlton and Squire 1993). Specifically, each dot pattern was a "high distortion" of a single underlying prototype dot pattern. Patterns were presented for $5 \mathrm{sec}$, and volunteers imagined pointing to the center dot in the pattern to guarantee attention. Actual pointing occurred in previous behavioral studies, but here no pointing was done in order to reduce movement in the scanner. Volunteers were not informed of the existence of a prototype.

After a short delay ( $\sim 2 \mathrm{~min})$, volunteers were told that the patterns had all belonged to a single category of patterns in the same sense that if a series of dogs had been presented, they would all belong to the category "dog." Scanning then occurred while a categorization test consisting of 72 novel dot patterns was presented. The test stimuli included 4 presentations of the prototype dot pattern $(\mathrm{P}), 16$ low distortions of the prototype dot pattern $(\mathrm{L}), 16$ high-distortion dot patterns $(\mathrm{H})$, and 36 unrelated, noncategorical patterns (U) (Fig. 1). The noncategorical patterns were derived from a different, unfamiliar, prototype and included 4 instances of this prototype, 16 low distortions, and 16 high distortions. The categorical patterns (the targets) and the noncategorical patterns (the foils) were similarly derived from prototype dot patterns. Accordingly, the test items differed only in that the categorical study items were examples of the learned category, whereas the noncategorical study items were examples of a different, unfamiliar category.

For each pattern, volunteers judged whether or not it came from the same category as the training patterns (a "yes" response was correct for the $\mathrm{P}$, L, and H patterns; a "no" response was correct for the U patterns). Each pattern was presented for $3.5 \mathrm{sec}$ with a 500-msec inter-item interval. The 72 test patterns were presented in eight blocks of 9 patterns each, which alternated between blocks containing predominantly categorical patterns and blocks containing predominantly random patterns.

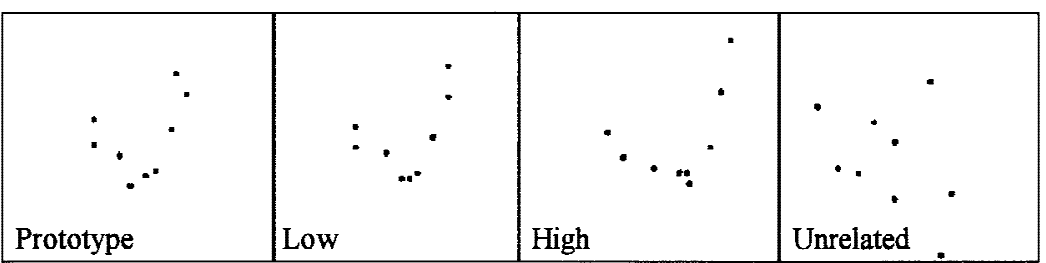

Figure 1: Examples of a prototype dot pattern $(\mathrm{P})$, low $(\mathrm{L})$ and high $(\mathrm{H})$ distortions of the prototype pattern, and an unrelated dot pattern $(U)$. In the categorization task, volunteers first viewed 40 high distortions of a single prototype pattern and imagined pointing to the center-most dot. At test, volunteers saw 72 novel dot patterns (4 presentations of the prototype, 16 low distortions of the prototype, 16 high distortions, and 36 unrelated dot patterns), and they judged whether each pattern was a member of the studied category. In the recognition task, volunteers viewed 40 dot patterns comprised of 8 repetitions of 4 different patterns. At test, 72 dot patterns were presented: 36 presentations of the study patterns (old) and 36 new dot patterns (new). Volunteers judged whether each item was a target.

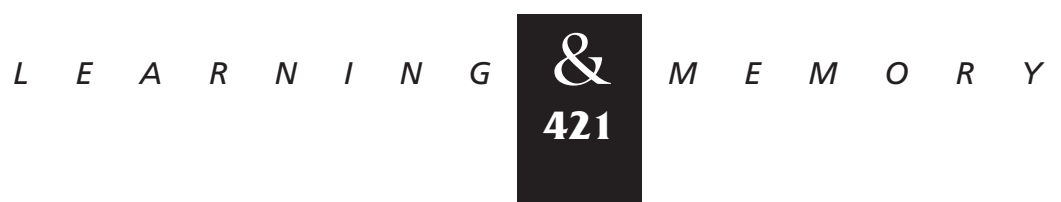




\section{Reber et al.}

Specifically, each block contained either seven categorical patterns that required a yes response and two random patterns that required a no response or vice versa. Volunteers made their yes-no categorical judgments using a fiber-optic button box. The entire study-test sequence was then repeated with different study items and test items (with 10 min between runs).

\section{RECOGNITION}

Ten volunteers studied 40 dot patterns one at a time. The 40 patterns consisted of 8 repetitions of each of 5 target items. In all other respects, this study phase was identical to the categorization test. After a short ( $2 \mathrm{~min})$ delay, scanning occurred while volunteers took a 72 -item recognition memory test involving 36 novel patterns and 36 target patterns (each of the 5 dot patterns that had been presented during study occurred either seven or eight times). Each pattern was presented for 3.5 sec with a 500-msec inter-item interval. Volunteers were instructed to use the button box to respond "yes" if the presented pattern had been seen during study and "no" if the pattern was novel. The patterns were presented in eight blocks of nine patterns each, which alternated between blocks containing seven old patterns and two new patterns and blocks containing seven new patterns and two old patterns. The entire study-test sequence was then repeated with different study and test items (with $\sim 10$ min between runs).

\section{FUNCTIONAL IMAGING}

Imaging was performed on a GE 1.5T SIGNA clinical MRI scanner fitted with a high-performance local head gradient and RF coils (Wong et al. 1991). Functional T2*-weighted images were acquired using an echoplanar single-shot pulse sequence with a matrix size of $64 \times 64$, echo time (TE) of $40 \mathrm{msec}$, flip angle of $90^{\circ}$, and in-plane resolution of $2.5 \times 2.5 \mathrm{~mm}$. For each scanning run, 82 images were acquired for each of 20-22 coronal slices ( $7 \mathrm{~mm}$ thick, 1-mm interslice gap) in an interleaved fashion with a repetition time (TR) of 3.6 sec. The first two images from each slice were discarded to assure that the MR signal had reached equilibrium on each slice. For anatomical localization, a standard whole-brain, T1-weighted, threedimensional MP-RAGE sequence was acquired (flip angle $=10^{\circ}, \mathrm{FOV}=24 \mathrm{~cm}, 256 \times 256 \times 128$ acqui- sition matrix; coronal slices, thickness $=1.5-1.6$ $\mathrm{mm})$.

Images were first corrected for distortion owing to field inhomogeneity (Reber et al. 1998b) and were coregistered through time using a two-dimensional registration algorithm (Cox 1996). Each slice was spatially smoothed using a two-dimensional (in-plane) gaussian kernel, FWHM = $7.5 \mathrm{~mm}$. Linear drift in the overall magnitude of the MR signal in each voxel over the course of the entire scan was eliminated (linear drift was estimated by computing the change in signal across the blocks that contained nontarget patterns). Within each run, voxels were eliminated if the signal magnitude changed $>10 \%$ between two samples $(3.6 \mathrm{sec})$ or if their mean signal level was below a threshold defined by the inherent noise in the data acquisition. Finally, the 36 runs were transformed (Collins et al. 1994) to conform to the atlas of Talairach and Tournoux (1988) with a final voxel size of 2.5 $\mathrm{mm}^{3}$. The 16 runs from the 8 volunteers who received the categorization task were combined (averaged), and the 20 runs from the 10 volunteers who received the recognition task were also combined.

Areas exhibiting activity selective for either the categorical or noncategorical dot patterns were identified by correlating the observed time course of activity in each voxel against an idealized reference function derived from the eight alternating blocks of dot patterns. The reference function was adjusted to reflect the lag between neural activity change and hemodynamic response (signal rise was assumed to occur linearly over a 6-sec delay; fall time was assumed to be linear over $9 \mathrm{sec}$ ). The resultant statistical map was then thresholded to eliminate voxels for which the correlation with the reference function was $<0.40\left(P<6 \times 10^{-5}\right.$, uncorrected for multiple comparisons). Furthermore, significant areas of activation were required to comprise a cluster of correlated voxels with a total volume $>350 \mathrm{~mm}^{3}$, corresponding to at least 22 contiguous voxels in the transformed data. Finally, clusters were required to be located in grey matter tissue.

\section{Results}

BEHAVIORAL

In the categorization condition, volunteers successfully learned about category membership from studying the high distortions. They endorsed

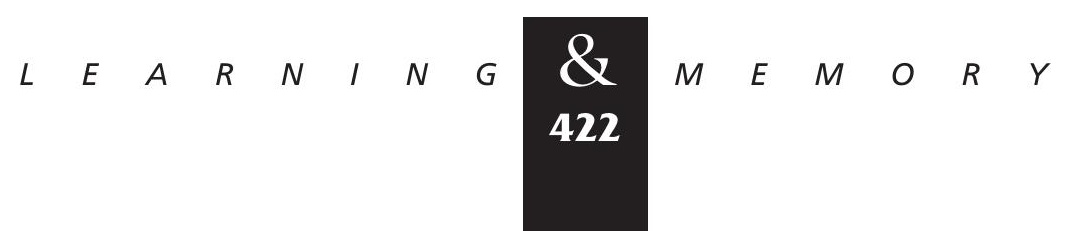


$71 \%$ of the prototypes, $55 \%$ of the low distortions, $54 \%$ of the high distortions, and $40 \%$ of the unrelated patterns (Fig. 2a). Overall categorization judgments were $58 \%$ correct, significantly better than chance $[50 \%, t(7)=4.5, P<0.005]$ and there was a significant effect of item type on endorsement rate $[F(1,3)=4.5, P<0.05]$. In the recognition condition, volunteers successfully learned the dot patterns presented at study (Fig. 2b). They were more likely to endorse the old dot patterns (81\%) than the new dot patterns (13\%) during the recognition test $[t(6)=9.7, P<0.001]$.

fMRI

Figure 3 shows the fMRI data identifying the brain areas in which significant stimulus-dependent changes in activity were observed for each task. For the categorization task (Fig. 3, top), the only areas of reliable change in activity occurred in posterior occipital cortex (BA 17/18). These areas exhibited reduced activity during judgments of categorical patterns relative to noncategorical patterns. Posterior occipital cortex was also the most prominent site of activity change in an earlier study of dot pattern category learning (Reber et al. 1998a).

For the recognition memory task (Fig. 3, bottom), a number of areas exhibited increased activity in response to the old patterns as compared with the new patterns. In particular, increased activity was observed in right occipital cortex (BA $17 / 18)$. The difference in brain activity in posterior
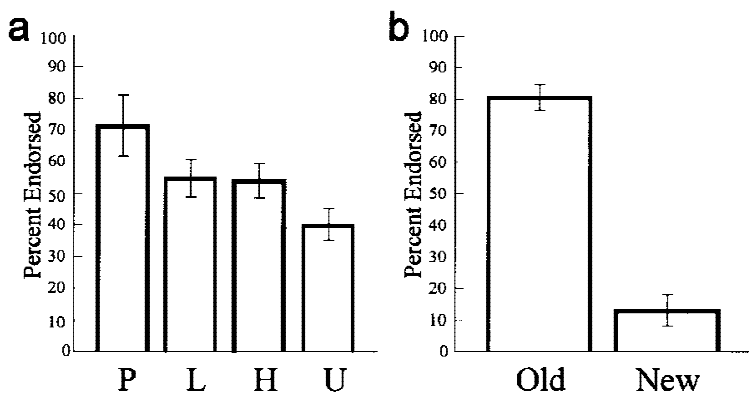

Figure 2: (a) Categorization performance. At test, the endorsement rate corresponded to how closely the patterns resembled the prototype of the training patterns (prototype, 71\%; low distortions, 55\%; high distortions, 54\%; unrelated patterns, 40\%). (b) Recognition memory performance. At test, volunteers were more likely to endorse the old items (81\%) than the new items (13\%) as having been studied previously. visual cortex (BA 17/18) between the categorization task and the recognition memory task is particularly striking (Fig. 4). During categorization, a sizeable decrease in activity was observed in occipital cortex that was similar to decreases reported previously in association with perceptual priming tasks (Squire et al. 1992; Schacter and Buckner 1998; Wiggs and Martin 1998). In contrast, during recognition, an increase in activity occurred in this region. The specific occipital areas exhibiting these changes were not identical in the two tasks. Accordingly, we performed additional analyses to examine the changes in activity that occurred in each of these areas in each task.

\section{CROSS-TASK ROI ANALYSES}

To contrast the changes in activity exhibited in specific areas of occipital cortex (BA 17/18) for both tasks, we defined three regions of interest (ROIs) in occipital cortex where stimulus-related (targets minus foils) changes in activity occurred in each task: two areas of decreased activity during categorization and one area of increased activity during recognition. Within these ROIs, we examined the changes in activity using a more lenient cluster-based threshold than in the first analysis (volume $\geqslant 150 \mu \mathrm{l}, r>0.22, P<0.05$ uncorrected). In addition, within each ROI, the percentage of voxels exhibiting a positive correlation with the reference function was calculated for each volunteer (number of voxels with $r>0.01$ divided by the total number of voxels in the ROI for each subject). Using these methods we found that during the recognition task there was increased activity in the same loci that had exhibited decreased activity in the categorization task. Specifically, we found increased activity in a 156- $\mu$ l subcluster in the more superior of the two posterior ROIs that had been identified from the categorization task (Fig. 4a). Also, for these two posterior ROIs, 60\% $( \pm 7.6 \%)$ of the voxels exhibited increased activity (old vs. new dot patterns), whereas during the categorization task (categorical stimuli vs. noncategorical dot patterns), only $14 \%( \pm 7.3 \%)$ of the voxels exhibited increased activity $[F(1,14)=9.0$, $P<0.01]$. Thus, there was no evidence for decreased activity in visual cortex for familiar stimuli during the recognition task.

The same analyses were then applied to the data from the categorization task within the posterior ROI that had exhibited increased activity in the recognition task. In the categorization task, this

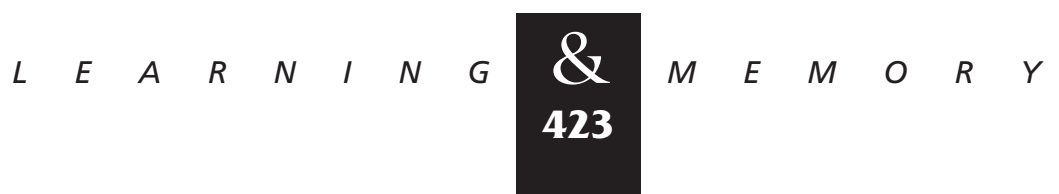




\section{Reber et al.}

Figure 3: Areas of significant task-related signal change are shown as color overlays on the averaged axial structural images [transformed to the atlas of Talairach and Tournoux (1988)]. The distance of each image relative to the AC-PC line $(-12$ to $37 \mathrm{~mm})$ is indicated for each image. Images are oriented according to radiologic convention with the right side of the brain on the left side of the image and anterior at the top. Areas where activation was greater when processing targets relative to foils are shown in red and yellow. In the categorization task, the targets were instances of the studied category, and in the recognition task, the targets were the recently studied items. Areas where activation decreased are shown in blue.

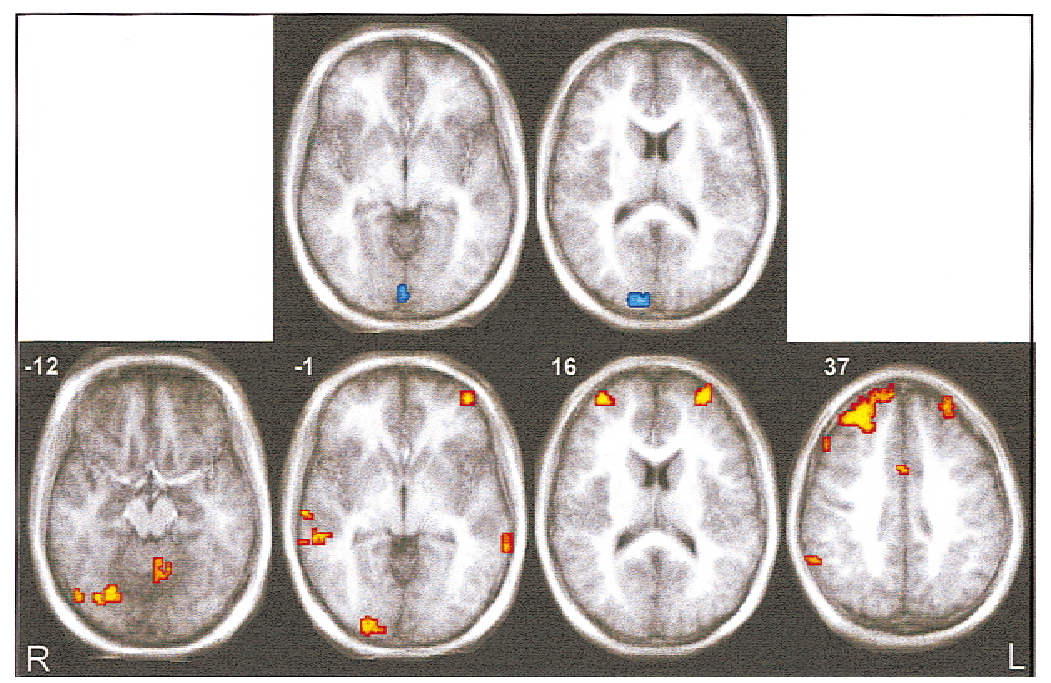
(Top) fMRI data for the categorization task. Two ROIs in posterior occipital cortex (BA 17/18; V1/V2) passed our statistical criteria (see text). Both areas exhibited reduced activity (blue) during the processing of categorical dot patterns relative to unrelated dot patterns. The ROIs were centered at Talairach coordinates $(x, y, z):(1,-88,-4)$ and $(12,-93,17)$. (Bottom) fMRI data for the recognition task. Fourteen ROls passed our criteria. There was significant activation in visual cortex in areas 17/18, V1/V2 (22, -93, -2) and in an ROI that included both occipital cortex (BA 19; 25, -72, -11) and cerebellum $(35,-80,-27)$. In addition, there were six frontal activations (i.e., greater activity while processing old items vs. new items, shown in red): right BA 6 , superior $(3,10,44)$ and middle $(8,17,65$; not shown) frontal gyri; bilateral area 9, prefrontal cortex $(29,49,34)$ and $(-31$, $55,20)$; right area 8, frontal eye fields (45, 20, 48); and left area 10, prefrontal cortex (-16, 62, -8 ; not shown). Three temporal lobe areas exhibited increased activity: bilateral area 21, middle temporal gyrus $(59,-36,2),(-67,-36,4)$, and $(58,-19,-6)$. One additional cerebellar activation was observed having two foci: $[(-7,-55,-14)$ and $(35,-72,-18)]$. Finally, there were two deactivations in left area 2, postcentral gyrus $(-47,-21,55$; not shown) and in left area 7 , posterior parietal cortex $(-39,-36,58$; not shown) that likely reflect the fact that volunteers usually made their no responses to new stimuli with the right hand.

area exhibited a weak tendency to be active (Fig. 4b). Specifically, increased activity was observed in a 156- $\mu$ l subcluster. Overall, in this ROI, 58\% $( \pm 16.3 \%)$ of the voxels exhibited increased activity during the categorization task. In contrast, in the recognition task, 95\% $( \pm 3.2 \%)$ of the voxels exhibited increased activity $[F(1,15)=7.7, P<0.05]$. Thus, some "recognition-like" activity may have occurred during the categorization task.

\section{Discussion}

The key finding was that when category judgments were made about dot patterns that resembled the dot patterns that had been presented for study, robust reductions in activity occurred in posterior occipital cortex. Yet, when recognition memory judgments were made for repeated and familiar dot patterns, activity increased in posterior occipital cortex. It appears that the act of engaging in a recognition memory task changes the way test stimuli are processed such that during recognition, stimuli are processed in a fundamentally different way than during categorization. During recognition, increased occipital activity for familiar stimuli appears to override decreases that are associated with nondeclarative memory. This effect could be owing either to the effort applied to making a recognition memory judgment or to the successful retrieval of a previously encountered stimulus.

This interpretation must be tempered by the fact that the two experimental conditions were not completely parallel. Despite matching the conditions as closely as possible, there were necessarily several differences between them. In the categorization condition, each pattern presented at study was a novel distortion of a single prototype dot pattern. In contrast, in the recognition condition, five patterns were each repeated eight times at study. Therefore, the tasks differed both in the number of times each dot pattern was repeated and in the similarity between the dot patterns in the study phase. Furthermore, in the test phase,

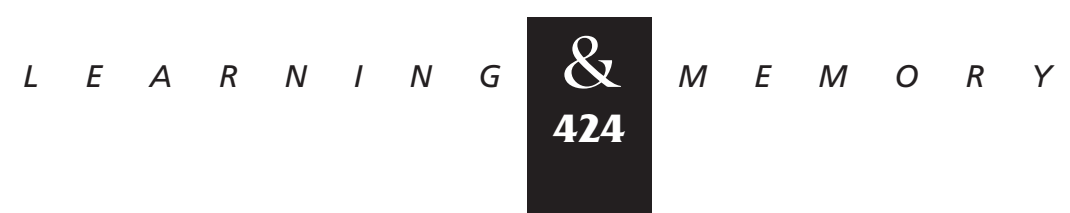




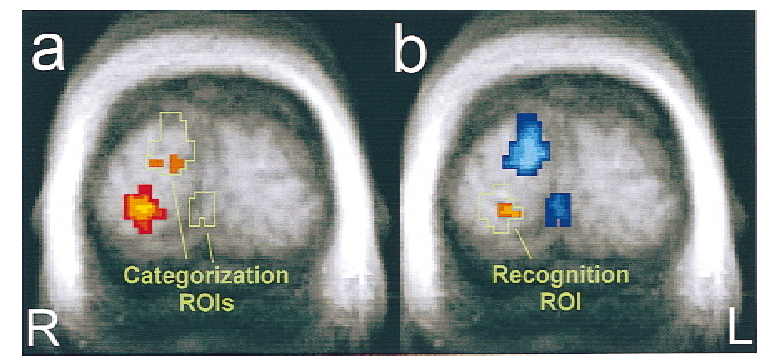

Figure 4: The posterior activity from the recognition task (a) and the categorization task (b) are shown here as colored overlays on the averaged coronal images at -93 $\mathrm{mm}$. Superimposed on the data from each task is the outline (green) of the posterior ROIs found in the other task. The voxels in these outlined regions were analyzed to determine how the posterior ROls behaved in the two tasks $(P<0.05$, uncorrected; $150-\mu l$ minimum cluster volume). (a) In the recognition task, a small subcluster of increased activity was found in one of the ROls (upper green outline) that had shown decreased activity in the categorization task. Thus, the same ROI that had decreased its activity during categorization increased its activity during recognition. (b) In the categorization task, a small subcluster of increased activity was found in the same posterior ROI (green outline) that had shown increased activity in the recognition task. Thus, some recognition-like activity also occurred during categorization.

the dot patterns used as foils in the categorization task were distortions of an unstudied prototype, created in the same manner as the target dot patterns. In the test phase of the recognition task, the dot patterns used as foils were random dot patterns with no inherent similarity to any other dot patterns.

For the present study, we chose not to use random dot patterns for the categorization task [as we did in our earlier study of categorization (Reber et al. 1998a)] in order to eliminate a potential confound. Specifically, when the noncategorical dot patterns in the categorization task are random dot patterns, it is possible that reduced visual cortical activity associated with categorical stimuli could be owing entirely to effects at the time of test (neighborhood effects based on the within-block similarity of the categorical dot patterns). This same potential confound existed in the current study in the case of the recognition task. However, in the case of recognition, using random dot patterns as foils and identical dot patterns as targets should, if anything, have made it more likely to observe a decrease in visual cortical activity for the target stimuli. Instead, we observed increased oc- cipital cortical activity for target stimuli during the recognition task.

Previous neuroimaging studies of perceptual priming have found decreased occipital activity for familiar stimuli (Squire et al. 1992; Buckner et al. 1995; Frith et al. 1995; Schacter et al. 1996; Backman et al. 1997). In these studies, decreased activity occurred in more anterior cortical areas (e.g., BA 19) than in our study (BA 17/18). The difference between our findings and previous reports could be attributable to either task differences (a categorization paradigm vs. a priming paradigm) or stimuli differences (dot patterns vs. word stems and pseudowords).

It should be noted that the changes in posterior occipital cortex observed during the categorization task (this paper; Reber et al. 1998a) are not the only areas involved in making categorization judgments. The analysis was based on contrasting the response to categorical and noncategorical dot patterns and therefore could not have identified brain areas that were responsive to both (such as areas used to make the categorization judgment itself). It should also be noted that three frontal cortical areas that were active in earlier work with this categorization task (Reber et al. 1998a) did not pass the statistical threshold in the current study. However, activity in one of these areas (left frontal cortex, BA 10) was detected with a slightly reduced statistical threshold $(r>0.35$ instead of $r>0.40$ ). The current study differed from the earlier one in that the noncategorical dot patterns were related to an unstudied prototype and thus had the same degree of relatedness to each other as did the categorical dot patterns. In the earlier study, the noncategorical dot patterns were random patterns.

During the recognition memory task, widespread increases in activity in response to old dot patterns were observed in the frontal and temporal lobes in addition to the occipital lobe. Recognition memory performance is known to require the medial temporal lobe (Reed and Squire 1997), and there have been reports of increased activity in the medial temporal lobe during recognition in comparison to a baseline condition (e.g., Fuji et al. 1997; Rugg et al. 1997). However, activation of this region has not been observed consistently during recognition tests (e.g., Nyberg et al. 1995; Rugg et al. 1996; see Schacter et al. 1996) and was not observed here. Schacter et al. (1995) found increased hippocampal activity in a recognition task that compared previously studied drawings of ob-

$$
\begin{array}{lllllllllllllll} 
& E & A & R & N & I & N & G & \begin{array}{l}
\boldsymbol{Q} \\
425
\end{array} & M & E & M & O & R & Y
\end{array}
$$




\section{Reber et al.}

jects and novel objects. The absence of task-related activity changes in the medial temporal lobe in our study may be owing to stimulus differences (objects vs. dot patterns) or to the fact that the memory demands when judging old and new items were more similar in our study than in the study by Schacter et al. (1995).

Neuroimaging studies of recognition memory that directly contrast the response to old and new stimuli have found increased activity bilaterally in prefrontal cortex (BA 10) (Rugg et al. 1996; Tulving et al. 1996). Our results are consistent with the idea that increased activity in the frontal lobe reflects recognition success and that the frontal lobe operates to integrate retrieved information about a stimulus and the encoding context (Squire et al. 1993). Previous studies of visual recognition memory have also reported increased occipital lobe activity in BA 17/18 [Nyberg et al. 1995; Rugg et al. 1996; Tulving et al. 1996; but note that Tulving et al. (1994) found a decrease in temporal lobe activity during recognition for auditory stimuli]. Thus, it appears that increased visual activity in response to familiar stimuli can be part of the recognition memory process. It should be noted that an earlier PET study that contrasted different measures of declarative memory and nondeclarative memory than measured here (cued recall and word-stem completion priming) found an overall decrease in activity in occipital cortex in both conditions (Squire et al. 1992). Because that study involved cued recall, not recognition, it is not clear what bearing it has on the suggestion that recognition is typically associated with increases in activity in posterior occipital cortex.

The lack of a priming-like effect in posterior occipital cortex during the recognition task is consistent with neuropsychological studies that have suggested that nondeclarative memory does not (or cannot) contribute to recognition memory judgments (Haist et al. 1992; Gabrieli et al. 1995; Reber and Squire 1998). In one compelling case, the severely amnesic patient E.P. performed at chance on recognition memory tasks but exhibited completely intact priming and category learning (Squire and Knowlton 1995; Hamann and Squire 1997). Thus, even though priming and category learning were intact, they could not be used to support recognition memory judgments. The neuroimaging findings presented here suggest an explanation for why priming and category knowledge (and perhaps other forms of nondeclarative memory) may not ordinarily support conscious judgments of familiarity. The process of making judgments of familiarity (recognition memory) establishes a brain state fundamentally different from the brain state achieved during nondeclarative memory tasks. As a result, reduced brain activity in association with the repeated, familiar stimuli [the hallmark of perceptual priming (see Schacter and Buckner 1998)] does not ordinarily occur during declarative memory tests. Declarative and nondeclarative memory appear to operate largely independently, and, for visual tasks, these two forms of memory can be distinguished by the contrasting patterns of activity in posterior visual cortex.

\section{Acknowledgments}

We thank R. Buxton and E. Wong for assistance with the functional imaging protocol, J. Moore for assistance in data collection, and J. Zouzounis. This research was supported by the Medical Research Service of the Department of Veterans Affairs, National Institute of Mental Health grant $\mathrm{MH} 24600$, the National Alliance for Research in Schizophrenia and Depression (NARSAD), an NIMH postdoctoral fellowship (F32 MH11150, P.J.R.), and McDonnell-Pew Center for Cognitive Neuroscience (C.E.L.S., P.J.R.).

The publication costs of this article were defrayed in part by payment of page charges. This article must therefore be hereby marked "advertisement" in accordance with 18 USC section 1734 solely to indicate this fact.

\section{References}

Backman, L., O. Almkvist, J. Andersson, A. Nordberg, B. Winblad, R. Reineck, and B. Langstrom. 1997. Brain activation in young and older adults during implicit and explicit retrieval. J. Cognit. Neurosci. 3: 378-391.

Buckner, R.L., S.E. Petersen, J.G. Ojemann, F.M. Miezin, L.R. Squire, and M.E. Raichle. 1995. Functional anatomical studies of explicit and implicit memory retrieval tasks. J. Neurosci. 15: 12-29.

Collins, D.L., P. Neelin, T.M. Peters, and A.C. Evans. 1994. Automatic three-dimensional inter-subject registration of MR volumetric data in standardized talairach space. J. Comput. Assisted Tomogr. 18: 192-205.

Cox, R.W. 1996. AFNI: Software for analysis and visualization of functional magnetic resonance neuroimages. Comput. Biomed. Res. 29: 162-173.

Frith, C.D., N. Kapur, K.J. Friston, P.F. Liddle, and R.S.J. Frackowiak. 1995. Regional cerebral activity associated with the incidental processing of pseudo-words. Hum. Brain Mapp. 3: 153-160.

$$
\begin{array}{lllllllllllllll} 
& E & A & R & N & I & N & G & \underset{4}{4} \\
426 & M & E & M & O & R & Y
\end{array}
$$


Fuji, T., J. Okuda, R. Kawashima, A. Yamadori, R. Fukatsu, K. Suzuki, M. Ito, R. Goto, and H. Fukuda 1997. Different roles of the left and right parahippocampal regions in verbal recognition: A PET study. NeuroReport 8: 1113-1117.

Gabrieli, J.D.E., D.A. Fleischman, M.M. Keane, S.L. Reminger, and F. Morrell. 1995. Double dissociation between memory systems underlying explicit and implicit memory in the human brain. Psychol. Sci. 6: 76-82.

Gardiner, J.M. and R.I. Java. 1993. Recognizing and remembering. In Theories of memory (ed. A. Collins, S. Gathercole, M. Conway, and P. Morris), pp. 163-188. Erlbaum, Hillsdale, NJ.

Haist, F., A.P. Shimamura, and L.R. Squire. 1992. On the relationship between recall and recognition memory. J. Exp. Psychol. Learn. Mem. \& Cognit. 18: 691-702.

Hamann, S.B. and L.R. Squire. 1997. Intact perceptual memory in the absence of conscious memory. Behav. Neurosci. 111: 850-854.

Jacoby, L.L. 1991. A process dissociation framework: Separating automatic and intentional uses of memory. J. Mem. Lang. 30: 513-541.

Jacoby, L.L. and M. Dallas. 1981. On the relationship between autobiographical memory and perceptual learning. J. Exp. Psychol. Gen. 3: 306-340.

Knowlton, B.J. and L.R. Squire. 1993. The learning of natural categories: Parallel memory systems for item memory and category-level knowledge. Science 262: 1747-1749.

Mandler, G. 1980. Recognizing: The judgment of previous occurrence. Psychol. Rev. 87: 252-271.

1989. Memory; Conscious and unconscious. In Memory: Interdisciplinary approaches (ed. P.R. Soloman, G.R. Goethals, C.M. Kelly, and B.R. Stephens), pp. 84-106. Springer-Verlag, New York, NY.

Nyberg, L., E. Tulving, R. Habib, L. Nilsson, S. Kapur, S. Houle, R. Cabeza, and A.R. McIntosh. 1995. Functional brain maps of retrieval mode and recovery of episodic information. NeuroReport 7: 249-252.

Posner, M.I. and S.W. Keele. 1968. On the genesis of abstract ideas. J. Exp. Psychol. 77: 353-363.

Reber, P.J. and L.R. Squire. 1998. Relaxing decision criteria does not improve recognition memory in amnesic patients. Mem. Cognit. (in press).

Reber, P.J., C.E.L. Stark, and L.R. Squire. 1998a. Cortical areas supporting category learning identified using fMRI. Proc. Natl. Acad. Sci. 95: 747-750.

Reber, P.J., E.C. Wong, R.B. Buxton, and L.R. Frank. 1998b. Correction of off-resonance related distortion in EPI using EPI based field maps. Magn. Reson. Med. 39: 328-330.
Reed, J.M. and L.R. Squire. 1997. Impaired recognition memory in patients with lesions limited to the hippocampal formation. Behav. Neurosci. 111: 667-675.

Rugg, M.D., P.C. Fletcher, C.D. Frith, M. Frambach, and R.J. Dolan. 1996. Differential activation of the prefrontal cortex in successful and unsuccessful memory retrieval. Brain 119: 2073-2083.

1997. Brain regions supporting intential and incidental memory: A PET study. NeuroReport 8: 1283-1287.

Schacter, D.L. 1994. Priming and multiple memory systems: Perceptual mechanisms of implicit memory. In Memory systems 1994 (ed. D.L. Schacter and E. Tulving), pp. 233-268. MIT Press, Cambridge, MA.

Schacter, D. and E. Tulving. 1994. Memory systems 1994 (ed. D.L. Schacter and E. Tulving). MIT Press, Cambridge, MA.

Schacter, D.L. and R.L. Buckner. 1998. Priming and the brain. Neuron 20: 185-195.

Schacter, D.L., E. Reiman, A. Ueker, M.R. Polster, L.S. Yun, and L.A. Cooper. 1995. Brain regions associated with retrieval of structurally coherent visual information. Nature 376: $587-590$.

Schacter, D.L., N.M. Alpert, C.R. Savage, S.L. Rauch, and M.S. Albert. 1996. Conscious recollection and the human hippocampal formation: Evidence from positron emission tomography. Proc. Natl. Acad. Sci. 93: 321-325.

Squire, L.R. 1982. The neuropsychology of human memory. Annu. Rev. Neurosci. 5: 241-273.

Squire, L.R. and B.J. Knowlton. 1995. Learning about categories in the absence of memory. Proc. Natl. Acad. Sci. 92: $12470-12474$

Squire, L.R., J.G. Ojemann, F.M. Miezin, S.E. Petersen, T.O. Videen, and M.E. Raichle. 1992. Activation of the hippocampus in normal humans: A functional anatomical study of memory. Proc. Natl. Acad. Sci. 89: 1837-1841.

Squire, L.R., B. Knowlton, and G. Musen. 1993. The structure and organization of memory. Annu. Rev. Psychol. 44: 453-495.

Talairach, J. and P. Tournoux. 1988. A co-planar stereotaxic atlas of the human brain. Thieme, Stuttgart, Germany.

Tulving, E., S. Kapur, H.J. Markowitsch, F.I. Craik, R. Habib, and S. Houle. 1994. Neuroanatomical correlates of retrieval in episodic memory: Auditory sentence recognition. Proc. Natl. Acad. Sci. 91: 2012-2015.

Tulving, E., H.J. Markowitsch, F. Craik, R. Habib, and S. Houle. 1996. Novelty and familiarity activations in PET studies of memory encoding and retrieval. Cereb. Cortex 6: 71-79.

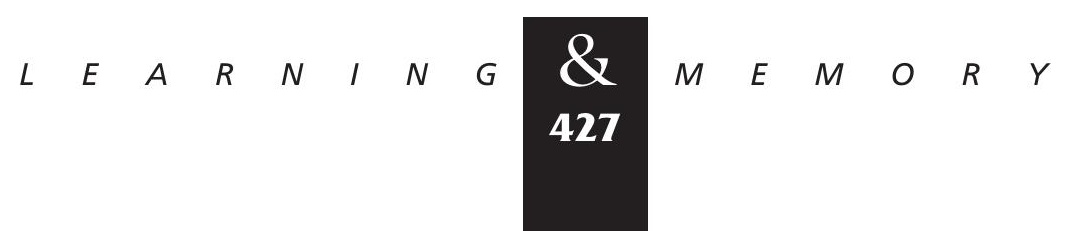




\section{Reber et al.}

Wagner, A.D., J.D.E. Gabrieli, and M. Verfaellie. 1997. Dissociations between familiarity processes in explicit recognition and implicit perceptual memory. J. Exp. Psychol. Learn. Mem. \& Cognit. 23: 305-323.

Weiskrantz, L. 1990. Problems of learning and memory: One or multiple memory systems? Phil. Trans. R. Soc. Lond. B. 329: $99-108$.

Wiggs, C.L. and A. Martin. 1998. Properties and mechanisms of perceptual priming. Curr. Opin. Neurobiol. 8: 227-233.

Wong, E.C., A. Jesmanowicz, and J.S. Hyde. 1991. Coil optimization for MRI by conjugate gradient descent. Mag. Res. Med. 21: 39-48.

Received July 7, 1998; accepted in revised form September 16, 1998. 


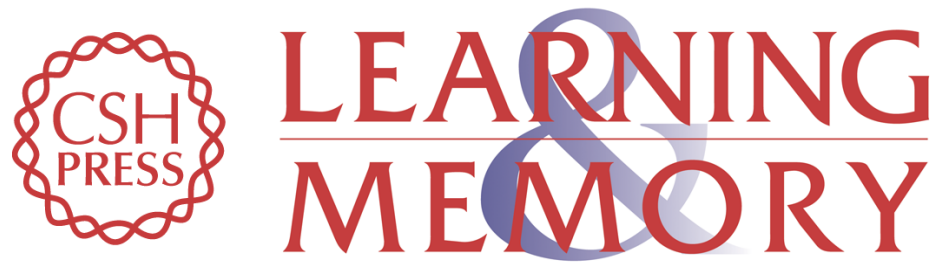

\section{Contrasting Cortical Activity Associated with Category Memory and Recognition Memory}

Paul J. Reber, Craig E.L. Stark and Larry R. Squire

Learn. Mem. 1998, 5:

Access the most recent version at doi:10.1101//m.5.6.420

References This article cites 30 articles, 7 of which can be accessed free at: http://learnmem.cshlp.org/content/5/6/420.full.htmI\#ref-list-1

License

Email Alerting Receive free email alerts when new articles cite this article - sign up in the box at the Service top right corner of the article or click here. 